\title{
Texture Analysis Using Pairwise Interaction Maps
}

\author{
Dmitry Chetverikov \\ Computer and Automation Research Institute \\ 1111 Budapest, Kende u.13-17, Hungary \\ email: mitya@leader.ipan.sztaki.hu
}

\begin{abstract}
Pairwise pixel interactions have proved to be a powerful tool in feature based $[3,7]$ as well model based $[9]$ texture analysis. Different aspects and components of the feature based interaction map (FBIM) approach have already been discussed, but no self-contained description of the FBIM has been published yet. This paper provides a comprehensive up-to-date survey of the approach, including major algorithms and a series of experimental studies that demonstrate the capabilities of the approach.
\end{abstract}

\section{Introduction}

Precise directional analysis is essential for the feature based interaction map approach proposed recently in $[3,7]$. Pattern orientation is viewed as direction of maximum statistical symmetry assessed via anisotropy. The term 'interaction map' was originally introduced in the Markov-Gibbs texture model with pairwise pixel interactions [9]. In feature based approach discussed here, this term refers to the structure of statistical pairwise pixel interactions evaluated through the spatial dependence of a texture feature computed from the extended graylevel difference histogram (EGLDH) introduced in [2]. The relation between the model based approach [9] and the feature based approach presented in this paper is discussed in [5].

Pairwise pixel interactions described by the EGLDH convey important structural information. Both short- and long-range interactions are relevant. Depending on the scale of the pattern, the range of spacings may include a short-range and a long-range zone. This distinction reflects the difference between the shortand the long-range order which is typical for natural patterns, e.g. crystals. For irregular patterns, the short-range zone is dominant, while structured patterns exhibit their regularity and orientation in the long-range zone.

The power of the FBIM approach is in its selectivity to the fundamental structural properties of textures, such as anisotropy, symmetry and regularity. The FBIM can be implemented as a running structural filter [4] that responds to the fundamental structural features of local pattern.

The FBIM approach has been successfully applied to a wide range of texture analysis tasks and applications $[3,7,8,4,6]$. These studies appeared in the proceedings of diverse conferences, each of them discussing certain aspects of the 
method. However, no self-contained, comprehensive description of the approach has ever been published. Such presentation is necessary for possible reproduction and use of the algorithms and the results by the members of the computer vision community.

In section 2, we present the structure and the major components of the FBIM approach to texture analysis. Section 3 shows how the fundamental structural features of textures can be recovered using the FBIM approach. Section 4 overviews the current applications of the approach. Finally, future plans and the limits of the approach are discussed.

\section{The FBIM approach}

The polar interaction map $M_{p l}(i, j)$ is the basic entity of the FBIM method. $M_{p l}(i, j)$ is an intensity-coded polar representation of an EGLDH feature, with the rows enumerating the angle $\alpha_{i}$, the columns the magnitude $d_{j}$ of the varying spacing vector. The main steps of the method are as follows.

Step 1 For a discrete set of spacing vectors $\boldsymbol{d}_{i j}=\left(\alpha_{i}, d_{j}\right), \alpha_{i} \in[0,2 \pi], i=$ $0, \ldots, N_{a}, d_{j} \in\left[1, d_{\text {max }}\right], j=0, \ldots, N_{d}$, compute an EGLDH feature $F\left(\alpha_{i}, d_{j}\right), N_{a}$ is the number of angles, $N_{d}$ the number of displacements.

Step 2 Define polar FBIM as $M_{p l}(i, j)=F\left(\alpha_{i}, d_{j}\right)$. Each column of $M_{p l}(i, j)$ is treated as a cyclical array and called anisotropy indicatrix describing texture anisotropy at the distance $d_{j}: A^{j}(i)=M_{p l}(i, j)$.

Step 3 Apply a reflectional symmetry transform to each indicatrix $A^{j}(i), j=$ $1, \ldots, N_{d}$, to obtain the symmetry indicatrix $S^{j}(i)$.

Step 4 Define polar symmetry map as $S_{p l}(i, j)=S^{j}(i)$.

Step 5 Compute the row projections of $S_{p l}(i, j)$ to find the directions of dominant symmetry and evaluate pattern anisotropy and orientation.

Step 6 Transform $M_{p l}(i, j)$ to $X Y$ coordinates to obtain the Cartesian $(X Y)$ interaction map $M_{x y}(k, l)$.

Step 7 Use $M_{x y}(k, l)$ to analyze texture structure.

\subsection{Computing the interaction maps}

The extended graylevel difference histogram shows the frequencies of the absolute graylevel differences between pixels separated by a spacing vector. In the EGLDH, the magnitude and the angle of the spacing vector are independent, arbitrary parameters, while in the conventional graylevel difference histogram (GLDH, e.g., [10]) these parameters are interrelated because of the image raster.

The computation of an EGLDH feature and the layout of $M_{p l}(i, j)$ are illustrated in figure 1. In figure 1a, $(m, n)$ scans the pixels of the image, while $(x, y)$ points at a non-integer location specified by the spacing vector. The intensity $I(x, y)$ is obtained by the linear interpolation of the four neighboring pixels, then truncated to integer. The absolute graylevel difference $|I(m, n)-I(x, y)|$ is used to address and increment a bin of the EGLDH. 
The standard GLDH features [10] can be used with the EGLDH as well. In this study, the EGLDH feature $F$ is usually the median of $|I(m, n)-I(x, y)|$. For structural filtering, the mean value of the absolute difference is used as an additive feature suitable for run filtering. Using the more robust median feature results in structurally similar, but somewhat more contrast interaction maps. Examples of median based interaction maps are-shown in figure 5.

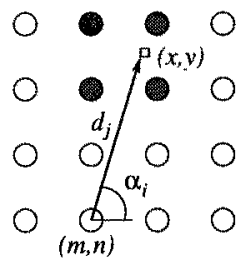

(a)

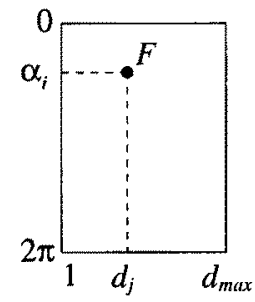

(b)

Fig. 1. (a) Computing the EGLDH. (b) The layout of the polar interaction map. $F$ is an intensity-coded EGLDH feature.

\subsection{Computing the symmetry map}

The anisotropy indicatrix $A^{j}(i)[2]$ is a column of $M_{p l}(i, j)$ treated as a cyclical array. The indicatrix exhibits texture anisotropy measured at a certain displacement $d_{j}$. While the map is intensity-coded, the indicatrix is represented by a polar diagram with the radius proportional to the EGLDH feature value. This diagram is used to compute the symmetry map from $M_{p l}(i, j)$. Figure 2 shows anisotropy and symmetry indicatrices computed for a regular and a linear structure.
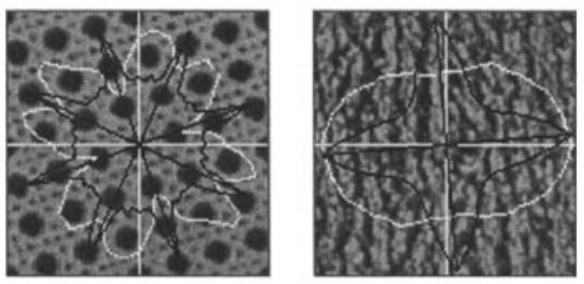

Fig. 2. Anisotropy (light) and symmetry (dark) indicatrices computed for a regular and a linear pattern. For the regular pattern, the displacement $d=22$ is close to the period. For the linear pattern, a small displacement $d=2$ indicates the anisotropy of the pattern. 
The symmetry map is composed of the columns that are symmetry indicatrices. Each symmetry indicatrix is obtained as a reflectional symmetry transform of the corresponding anisotropy indicatrix. For these purpose, a symmetry value is assigned to each direction $\alpha_{i}$, as illustrated in figure 3 . The two halves of the anisotropy indicatrix $A(i)$ are matched to yield the symmetry indicatrix $S(i)$.

The symmetry indicatrix $S(i)$ is defined as follows:

$$
S(i)=\left(\frac{2}{i_{\max }} \sum_{k=1}^{i_{\max } / 2}\left[1-\left|\frac{A(i+k)-A(i-k)}{A(i+k)+A(i-k)}\right|\right]\right)^{\gamma}
$$

Here $i=1, \ldots, i_{\max }$, where $i_{\max }=N_{a}-1 ; k$ is defined $\left(\bmod i_{\max }\right)$. The power $\gamma=5$ is used to make the symmetry measure more sensitive.

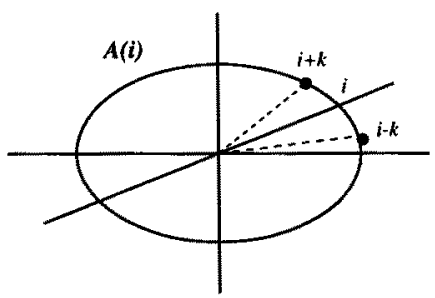

Fig. 3. Computing reflectional symmetry of the anisotropy indicatrix $A(i)$ for the angle $\alpha_{i}$.

\subsection{Analyzing texture symmetry and orientation}

An algorithm for analysis of the symmetry map was proposed in [3]. The analysis is aimed at the evaluation of pattern anisotropy and orientation defined by the axes of maximum symmetry. This is done for the short- and the long-range zones separately. For regular patterns, the short-range zone is not specific. For irregular pattern, this zone is relevant, while the decorrelated long-range zone only distorts the results by adding high but non-informative symmetry values. The procedure [3] tries to separate the two zones in the symmetry map. The border between the zones is detected as the minimum correlation between pairs of columns separated by a small displacement. The operation of this algorithm is illustrated in figure 4 .

Once the zones have been identified, the orientation analysis in each zone involves computing the row projections for each zone separately. The directions of the maximum symmetry are the peaks of the projections. These directions indicate the orientation of texture. In figure 4 , the row projections are shown as polar diagrams overlaid on the pattern. 


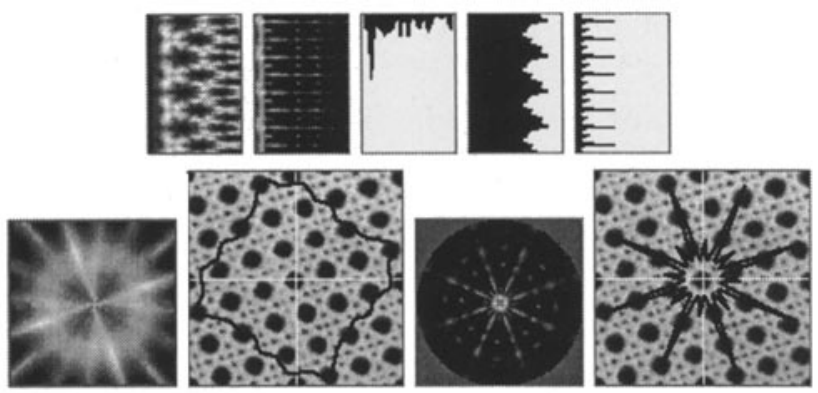

Fig. 4. Computing anisotropy and orientation of a texture pattern. Upper row: polar interaction map; polar symmetry map; correlations between columns of symmetry map; row projections of short-range zone; row projections of long-range zone. Lower row: short-range symmetry map; short-range anisotropy; long-range symmetry map; longrange anisotropy.

\section{Recovering the basic structural features of textures}

Figures 5 exemplifies the FBIM analysis of different natural textures from the album [1]. The first pattern is an irregular linear structure. The rest of the textures are structured patterns with various degrees of regularity. For the first pattern the short-range zones of $M_{x y}(k, l)$ is displayed enlarged. The structures of the other patterns are represented by the dark blobs of the interaction maps.
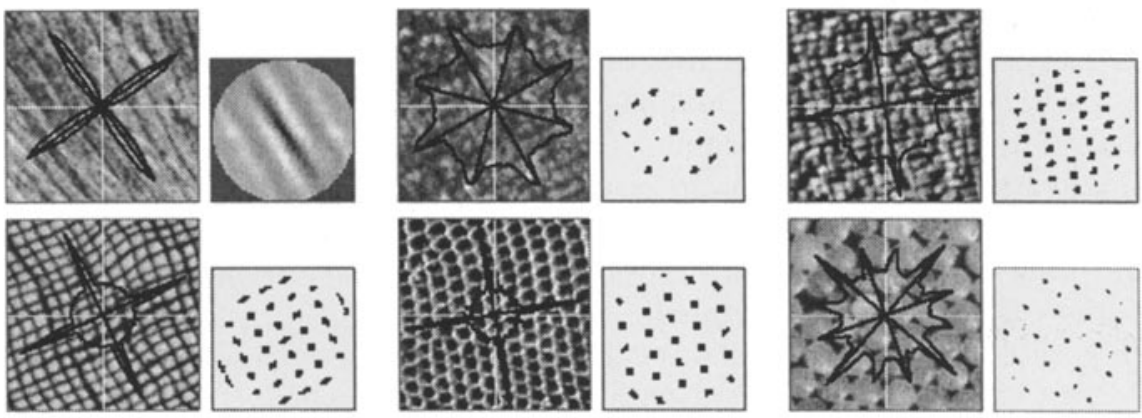

Fig. 5. Texture patterns with anisotropies overlaid and structures shown separately.

The anisotropies, orientations and structures of the patterns are correctly indicated even for weak structures. The texture orientation results by the FBIM approach were compared in [3] to the results obtained by an alternative, Fourier based method. The superiority of the FBIM over the spectral domain was further discussed in [4]. 


\section{Applications of the FBIM approach}

\subsection{Rotation-invariant texture classification}

To demonstrate the texture discriminating power of the FBIM approach, rotation-invariant texture classification experiments were carried out in [7]. Thirty textures were selected and digitized to $512 \times 512 \times 8$ resolution test images.

The test set contained patterns with different degree of regularity as well as non-regular directional patterns. No isotropic irregular textures were included since they do not have characteristic interaction maps suitable for discrimination. This is a limitation of the proposed approach as far as classification is concerned. Another condition is a relatively large size of the texture patches to be classified: the patch size should be large enough to contain two or more periods of any structured texture included in the test set. Otherwise, the interaction map does not represent the structure of the pattern.

Under these conditions, an accuracy of $96 \%$ was achieved using a simple but strict classification procedure that assigns to the test patch the class of the learning sample whose map is most similar to the map of the test patch.

\subsection{Using the FBIM filter to detect texture defects}

Detection of structural imperfections in textures is a specific task of texture analysis related to automated inspection of materials and surfaces. Recently, we have proposed a uniform approach to texture defect detection based on the concept of structural filtering introduced in [4]. When the polar maps of a reference sample and a sliding window are matched, $M_{p l}(i, j)$ becomes a powerful structural filter selective to anisotropy and regularity. Both rotation and scaling are easily incorporated into $M_{p l}(i, j)$ making the polar map suitable for orientation- and size-adaptive filtering. Potential applications and limits of the FBIM filter were discussed in [4] where a fast running implementation of the filter was proposed.

Many natural textures contain gradual variations of orientation and size that may be treated as tolerable or intolerable depending on the application. The FBIM filter can cope with both types of variations by specifying the degrees of variations that should be tolerated.

Figure 6 shows the four $256 \times 256$ pixel size grayscale test images used in the defect detection experiments, with sample detection results overlaid. Each of the images contains a single structural imperfection. The defect detection tests were run for different ranges of the algorithm parameters. More details of this experimental study are given in a forthcoming paper [6].

\subsection{Applications to document analysis}

A typical problem of document image analysis is the text/non-text separation. In a recent study [8], we applied the FBIM approach to the problem of zone classification in document image processing. Document blocks were labeled as 

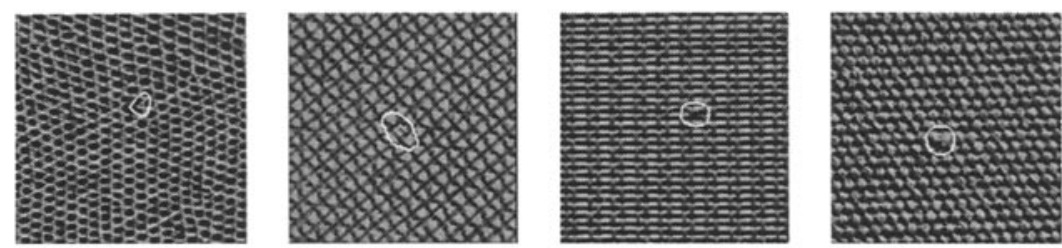

Fig. 6. The test textures with sample detection results overlaid.

text or non-text using texture features derived from a feature based interaction map.

The zone classification method proposed was tested on the comprehensive document image database UW-I created at the University of Washington in Seattle. This database comprises about 1000 digitized pages with approximately 15000 zones. Different classification procedures were considered. The performance ranged from $96 \%$ to $98 \%$ using 6 FBIM texture features only.

Another application is the determination of the document skew for subsequent skew normalization. The skew angle was computed as the orientation of the texture composed of the text lines. The original resolution of the UW-I document images was reduced by a factor of 8 . The angular resolution of the FBIM was $0.5^{\circ}$. The row projection array of the symmetry map was interpolated to calculate more accurate position of the maximum. The probability that the estimated skew angle lies within $0.5^{\circ}$ from the ground truth was $96 \%$.

\section{Conclusion}

FBIM is an efficient tool for texture analysis whose capability to solve different tasks has been demonstrated by numerous experiments in various applications. Currently, a basic limitation of the approach seems to be its unsatisfactory performance in the case of irregular patterns whose interaction maps do not show specific structure. We are now investigating this problem in order to find a solution based on a more detailed analysis of the short-range zone of the maps.

A related open problem is that of finding textured regions in images and roughly classifying the regions as irregular, linear, or structured. Interaction maps of each category have specific features that should be detected and analyzed. When the rough classification is done, the structural analysis should proceed depending on the category.

Other plans for future research and application of the FBIM approach include creation of a local symmetry filter, invariant detection of textured objects, shape from texture and query-by-texture in image databases. 


\section{Acknowledgment}

This work was supported in part by the grants OTKA T14520 and EU INFO COPERNICUS IC15 CT94 0742.

\section{References}

1. P. Brodatz. Textures: a photographic album for artists and designers. Dover, New York, 1966.

2. D. Chetverikov. GLDH based analysis of texture anisotropy and symmetry: an experimental study. In Proc. International Conf. on Pattern Recognition, pages 444-448. Vol.I, 1994.

3. D. Chetverikov. Pattern orientation and texture symmetry. In Computer Analysis of Images and Patterns, pages 222-229. Springer Lecture Notes in Computer Science vol.970, 1995.

4. D. Chetverikov. Structural filtering with texture feature based interaction maps: Fast algorithm and applications. In Proc. International Conf. on Pattern Recognition, pages 795-799. Vol.II, 1996.

5. D. Chetverikov. Texture feature based interaction maps: Potential and limits. In Proc. of Seminar on Theoretical Foundations of Computer Vision, Dagstuhl, Germany, 1997 (to appear). Springer Verlag.

6. D. Chetverikov and K. Gede. Textures and structural defects. In International Conference on Computer Analysis of Images and Patterns, Kiel, Germany, 1997 (to appear).

7. D. Chetverikov and R.M. Haralick. Texture anisotropy, symmetry, regularity: Recovering structure from interaction maps. In Proc. British Machine Vision Conference, pages 57-66, 1995 .

8. D. Chetverikov, J. Liang, J. Kömüves, and R.M. Haralick. Zone classification using texture features. In Proc. International Conf. on Pattern Recognition, pages 676-680. Vol.III, 1996.

9. G. Gimel'farb. Non-Markov Gibbs texture models with multiple pairwise pixel interactions. In Proc. International Conf. on Pattern Recognition, pages 591-595. Vol.II, 1996.

10. R. M. Haralick and L. G. Shapiro. Computer and Robot Vision, volume I. AddisonWesley, 1992. 